\section{MS10-P3 Synthesis, X-ray structural study and theoretical investigation of new coordination complexes based on benzimidazole derivatives.}

Sofiane BOUACIDA
H. Merazig $^{1}$, S. Sahki²
A. Bouraiou H. Merazig ${ }^{2}$

1. Département sciences de la matière, université Oum El Bouaghi, 04000 Oum El Bouaghi Algeria.

2. Unité de Recherche de Chimie de l'Environnement et Moléculaire Structurale, Université des frères Mentouri. Constantine 25000, Algeria.

email: bouacida_sofiane@yahoo.fr

Benzimidazole derivatives are reported to be physiologically and pharmacologically active and have shown different biological activities such as antioxidant, antifungal, antitumoral, anti-inflammatory and antimicrobial. The ability of benzimidazole derivatives to form stable complexes with metal ions, had given a place to a variety of metal-ligand coordination modes. Several research teams have examined the coordination behavior of benzimidazole derivatives towards transition metal ions and others studies have explored the biological activity of coordination compounds containing benzimidazole entity.

A ligand, 2-( $1 H$-benzo $[d]$ imidazole-2-yl)quinoline and its Cobalt and Manganese complex have been synthesized. The molecular structure of ligand and its $\mathrm{Co}(\mathrm{II})$ and $\mathrm{Mn}$ (II) complex are determined by single crystal X-ray diffraction technique. We report here the synthesis, crystallographic study, hydrogen bond interactions and theoretical investigation of two new coordination complexes based on benzimidazole derivative. In order to provide a better understanding of the bonding and the electronic structure of the ligand and their neutral complexes, we have carried out DFT calculations. The optimized structures reproduce those observed experimentally, while the reduced ones undergo remarkable geometrical parameters.

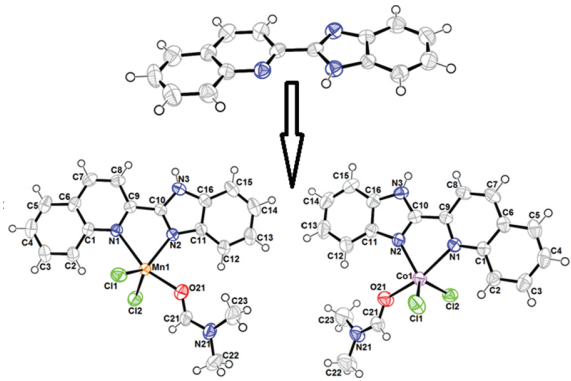

Figure 1. ORTEP of molecular structure of organic ligand and their coordination complexes.

Keywords: Benzimidazole derivatives, single crystal, hydrogen bond, theoretical investigation

\section{MS10-P4 Charge density studies and \\ topological analysis of X-H $\cdots \mathrm{S}$ hydrogen bonds}

Anita M. Grześkiewicz ${ }^{1}$, Maciej Kubicki ${ }^{2}$

1. Adam Mickiewicz University, Poznań, Poland 2. Adam Mickiewicz University, Poznań, Poland

email: aniow@amu.edu.pl

From many, many years hydrogen bonds - due to their great importance in biological systems - have been extensively studied in terms of geometry, energy and many others aspects. One of the method of studying hydrogen bond is Bader's quantum theory - Atoms in Molecules. This analysis permits to characterize interatomic interactions by means of the topological properties of charge density in the critical point. There are several articles that point out some relevant systematic relationship between the topological properties at the critical point and geometric parameters of $\mathrm{HB}$ [1-5]. However they mostly concern the $\mathrm{X}-\mathrm{H} \cdots \mathrm{O}$ interactions. We have decided to perform similar research in case of sulfur atom as an acceptor of different type of HBs. To do that we have determined the experimental charge density distribution in crystals of 4 different thioamides (2-mercaptobeznothiazole, 5-chloro-2mercaptobeznothiazole, thiobarbituric acid and 4-amino-6-hydroxy-pirimidine-2-thione), in order to obtain a good basis for determining the aforementioned dependence. The results of analysis will be presented in the communication.

References [1] Espinosa, E., Molins, E. \& Lecomte, C. Chemical Physics Letters 285, 170-173 (1998). [2] Espinosa, E., Souhassou, M., Lachekar, H. \& Lecomte, C. Acta Crystallographica Section B Structural Science 55, 563-572 (1999). [3] Espinosa, E., Lecomte, C. \& Molins, E. Chemical Physics Letters 300, 745-748 (1999). [4] Ranganathan, A., Kulkarni, G. U. \& Rao, C. N. R. Journal of Molecular Structure 656, 249-263 (2003). [5] Munshi, P. \& Row, T. N. G. CrystEngComm 7, 608-611 (2005).
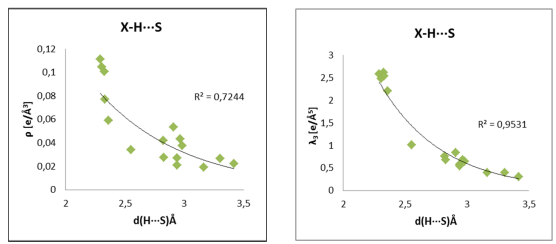

Figure 1. Exponential fitting of $\rho$ (electron density in the critical point) $\lambda$ (positive curvature of the electron density in the critical point) and against hydrogen $\cdots$ sulfur distance $[\mathrm{d}(\mathrm{H} \cdots \mathrm{S})]$

Keywords: weak hydrogen bonds, sulfur, charge density, topological analysis 